João Paulo Caldas Cardozo' Bianca Souza Lima ${ }^{2}$ Vinícius Zacarias Maldaner da Silva ${ }^{3}$ Gerson Cipriano Júnior ${ }^{4}$
* Recebido em:30/09/2011 Aprovado em: 08/03/2013

1 Graduando em Fisioterapia pelo Centro Universitário de Brasília (UniCEUB), Brasília/DF.

2 Prof. Membro do Programa de Mestrado em Educação Física da Faculdade de Educação Física da Universidade de Brasília (UnB), Brasília/DF.

3 Prof. Dr. Membro do Programa de Mestrado em Educação Física da Faculdade de Educação Física da Universidade de Brasília (UnB), Brasília/ DF. Orientador do PROIc - UnB.

4 Prof. Adjunto do Curso de Fisioterapia, Universidade de Brasília, UnB.

\section{Ajustes cardiovasculares frente às diferentes metodologias de exercício resistido em adultos saudáveis do sexo masculino*}

\section{Cardiovascular adjustments facing different methods of resistance exercise in healthy adult male}

\section{Resumo}

Os exercícios resistidos (ER) têm sido atualmente recomendados junto aos programas de atividade física como importante medida terapêutica adjuvante na melhora da capacidade cardiorrespiratória. O objetivo foi avaliar as alterações agudas no sistema cardiovascular frente às diferentes metodologias do exercício resistido em indivíduos saudáveis do sexo masculino. Estudo transversal com 13 indivíduos submetidos a 3 diferentes protocolos de ER: Concêntrico de extensores do joelho (A1); Concêntrico de flexores do joelho (A2) e Supersérie (A3). Para verificar o comportamento cardiovascular, foram mensuradas: pressão arterial (PA), frequência cardíaca (FC) de pico e variabilidade da frequência cardíaca (VFC). Houve aumento significativo da PA em todas as metodologias, sendo mais exacerbado em A1 e A3. A FC apresentou um caráter ascendente entre as séries de todas as metodologias. Essas mudanças apontam uma sobrecarga cardiovascular e uma supressão vagal diferenciada durante as diferentes metodologias de ER, sendo estas mais efetivas durante a A1.

Palavras-chave: Exercício resistido. Sistema cardiovascular. Variabilidade da frequência cardíaca. Sistema nervoso autônomo.

\begin{abstract}
The resistance exercises (RE) are currently recommended together physical activity programs as an important auxiliary therapeutic way to improve the cardiopulmonary capacity. The objective was to evaluate acute changes in the cardiovascular system face different methodologies of resistance exercise in healthy adult male. Cross-sectional study composed by 13 volunteers, submitted to 3 different protocols of RE: concentric knee extensors (A1); concentric knee flexors (A2) and super series exercise (A3). To verify the cardiovascular comportment, was measured: blood pressure (BP), heart rate (HR) and heart rate variability (HRV). A significative increment of BP was observed in all methodologies of RE, but A1 and A3 were more exacerbated. The HR showed an ascending behavior between the series of the 3 different forms of RE studied. These changes show us a cardiovascular overload and a vagal suppression differentiated during the different methodologies of RE, being these responses more pronounced during the A1 methodology.
\end{abstract}


Keywords: Resistance exercise. Cardiovascular system. Heart rate variability. Autonomic nervous system.

\section{Introdução}

O exercício resistido (ER) é capaz de provocar importantes alterações no funcionamento do sistema cardiovascular, levando a uma série de ajustes autonômicos com o objetivo de aumentar o fluxo sanguíneo local, atendendo, assim, ao aumento da demanda metabólica (NEVES et al., 2006). O aparelho isocinético oferece diferentes metodologias de aplicação do ER, sendo que cada uma delas demanda do organismo diferentes necessidades metabólicas, resultando em diferentes respostas cardiovasculares (PASCHOAL et al., 2006).

Segundo Malfatti et al. (2006), os efeitos musculares do treinamento isocinético já são extremamente consolidados na literatura, entretanto, poucas pesquisas se concentram na análise das demais respostas geradas por esse tipo de exercício. O isocinético oferece diferentes maneiras de realização do exercício resistido, no qual se pode sobrecarregar intercaladamente os músculos agonistas e antagonistas, ou concentrar a carga primariamente em um deles (FLECK; KRAEMER, 2006). Consequentemente, cada metodologia demanda do organismo diferentes quantidades de energia, acarretando em diferentes respostas e adaptações cardiovasculares (FLECK; KRAEMER, 2006; NEVES et al., 2006).

A frequência cardíaca (FC) é normalmente utilizada como forma de controlar a intensidade das atividades (VIANNA et al., 2005). Segundo Polito e Farinatti (2003), durante o exercício, a FC se comporta de maneira ascendente, aumento esse mediado principalmente pelo aumento da demanda metabólica, pelo aumento da atividade simpática do coração e pela oclusão dos vasos por meio da contração muscular.

A variabilidade da frequência cardíaca (VFC) descreve as oscilações do tempo transcorrido entre duas ondas R consecutivas no eletrocardiograma, denominado intervalo R-R, que estão relacionadas às influências do sistema nervoso simpático (SNS) e sistema nervoso parassimpático (SNP) sobre o nó sinusal (CAMBRI et al., 2008; FREEMAN et al. 2006; VANDERLEI et al., 2009). Trata-se de uma importante ferramenta de avaliação não invasiva da integridade da função neurocardíaca de indi- víduos saudáveis, atletas e portadores de doenças (FREEMAN et al., 2006; NEVES et al., 2006; VANDERLEI et al., 2009).

A pressão arterial (PA) é a variável hemodinâmica referente à pressão exercida pelo fluxo sanguíneo sobre a superfície interna das artérias, decorrente da expulsão sanguínea durante a sístole ventricular (ASSIS; OLIVEIRA, 2003; POLITO; FARINATTI, 2003). A importância de sua mensuração reside no fato de averiguar o relativo estresse cardiovascular, por meio do consumo de oxigênio do miocárdio estimado pelo duplo-produto (POLITO; FARINATTI, 2003).

A prática regular de atividade física tem sido referida como um fator de incremento no tônus vagal devido às adaptações fisiológicas ocorridas pelo aumento do trabalho cardíaco, levando à diminuição da FC máxima e submáxima, aumento da VFC e, consequentemente, diminuição da PA e risco cardiovascular, sendo que essas adaptações variam de acordo com o exercício realizado e a intensidade com que são realizados (NEVES et al., 2006; VANDERLEI et al., 2009).

O presente estudo permitirá conhecer o diferencial de cada metodologia sobre o sistema cardiovascular quando aplicadas na população saudável, servindo de base para estratificá-las e determinar qual exige mais ou menos do sistema cardiovascular, sendo essa uma importante informação para estudos envolvendo populações com limitações nesse sistema.

Este estudo teve como objetivo avaliar as alterações agudas no sistema cardiovascular frente às diferentes metodologias do exercício resistido em indivíduos saudáveis do sexo masculino.

\section{Métodos}

O presente estudo foi aprovado pelo Comitê de Ética em Pesquisa do Centro Universitário de Brasília, em referendo à aprovação prévia pelo Comitê de Ética em Pesquisa do Instituto do Coração (IC-DF), Parecer, PIC 108/09.

O trabalho se caracteriza também como um estudo analítico transversal, onde, sob o mesmo protocolo, 13 indivíduos foram avaliados no Laboratório de Biomecânica da Faculdade de Educação Física da Universidade de 
Brasília, sob a supervisão da cardiologista Dra. Alexandra Lima, do ambulatório de cardiologia do hospital Instituto de Cardiologia do Distrito Federal (IC-DF).

Foram admitidos no estudo apenas indivíduos do sexo masculino, considerados fisicamente ativos pelo questionário International Physical Activity Questionnaire (IPAQ) versão curta, validado para língua portuguesa, com idades entre 18 e 35 anos, ausência de história atual ou prévia de doença, ou procedimento cirúrgico e ausência de tratamento clínico atual, ao passo que foram excluídos aqueles indivíduos considerados inativos pelo questionário IPAQ versão curta, ou que apresentaram alterações clínicas durante a avaliação inicial, tais como hipertensão, ausculta pulmonar ou cardíaca alterada.

O protocolo iniciou-se com uma avaliação clínica realizada por meio de questionário padronizado, seguido de avaliação ergoespirométrica realizada em esteira ergométrica (T2100, General Eletrics, EUA), com análise simultânea da ventilação pulmonar e gases expirados por meio de analisador metabólico (K4, Cosmed ${ }^{\circledR}$, Itália) e com registro eletrocardiográfico (Cardiosoft, General Eletrics, USA), a fim de viabilizar a participação do voluntário no estudo. Foi utilizado um protocolo de rampa, com incremento progressivo da intensidade e duração de 8 a 12 minutos, sendo sintoma limitante o cansaço do avaliado, em consonância com normas estipuladas pelo American College of Sports Medicine.

Posteriormente, foi entregue e explicado o Termo de Consentimento Livre e Esclarecido (TCLE) e o IPAQ versão curta, traduzido e validado para a língua portuguesa. O questionário IPAQ classifica um indivíduo quanto ao nível de atividade física, como: muito ativo (5), ativo (4), irregularmente ativo $\mathrm{A}$ (3), irregularmente ativo B (2) e sedentário (1).

Vale ressaltar que o TCLE foi entregue somente após avaliação clínica inicial, porque optamos por apresentá-lo apenas àqueles considerados aptos pela médica responsável a participar do estudo, e que todos os voluntários foram advertidos quanto à importância e aos riscos do exame ergoespirométrico contido nessa avaliação.

Após o consentimento, foi realizado o sorteio para aleatorização da sequência de aplicação das diferentes metodologias de exercício resistido (A1, A2 ou A3), sendo:
(A1) Exercício concêntrico do agonista isolado: exercício concêntrico dos extensores de joelho, em 3 séries de 10 repetições, com velocidade de $60^{\circ} / \mathrm{seg}$. e intervalo de recuperação de 60 segundos.

(A2) Exercício concêntrico do antagonista isolado: exercício concêntrico de flexores de joelho, em 3 séries de 10 repetições, com velocidade de $60^{\circ} / \mathrm{seg}$. e intervalo de recuperação de 60 segundos.

(A3) Exercício alternado ou supersérie: exercício concêntrico dos flexores do joelho seguido, após 10 segundos, do exercício concêntrico dos extensores do joelho, em 3 séries de 10 repetições com velocidade de $60 \%$ seg. e intervalo de 60 segundos entre cada série.

Os protocolos foram aplicados em dias separados, com intervalo de 7 (sete) dias entre eles, sempre no mesmo turno, isto é, aqueles que iniciaram o protocolo no período matutino, findaram-no nesse mesmo período. $\mathrm{O}$ mesmo procedimento é válido para os que optaram pelo período vespertino, sendo essa uma forma de padronizar as coletas.

Para aplicação das diferentes metodologias de exercício resistido, foi utilizado um dinamômetro isocinético (Biodex System 3, Biodex Medical, Shirley, NY), localizado no Laboratório de Força Muscular da Faculdade de Educação Física da Universidade de Brasília (LFM - FEF/UnB); e, para registro e estocagem dos dados, foi utilizado o programa Biodex Advantage versão 3.0.

Antes de cada modalidade, os voluntários realizaram um aquecimento em cicloergômetro (Corival, Lode BV, Holanda) com a carga de 50 Watts, durante 5 minutos. Após o aquecimento geral, foi realizado um específico, por meio de exercícios no próprio isocinético, com velocidade de $300 \%$ s, em 2 séries de 10 repetições.

Os voluntários foram posicionados na cadeira, fixados por meio de cintos de segurança no tronco, na pelve e na coxa, de modo a evitar movimentos e/ou compensações indesejadas que pudessem influenciar o resultado, com a possibilidade de um movimento livre e confortável de flexão e extensão do joelho. Nesse processo, a extensão completa do joelho foi definida como $0^{\circ}$ e a 
flexão a $90^{\circ}$, utilizando-se uma amplitude de movimento de flexoextensão de aproximadamente $90^{\circ}$. O epicôndilo lateral do fêmur foi usado como ponto de referência do eixo de rotação do joelho ao ser alinhado com o eixo de rotação do aparelho.

A posição inicial de teste foi estabelecida pela flexão a $90^{\circ}$ do joelho e, a partir dela, os indivíduos foram instruídos a realizar os movimentos de extensão e flexão do joelho do membro direito, em velocidade pré-estabelecida de $60 \%$ seg. O membro inferior direito sendo utilizado como padrão, pois estudos anteriores não demonstraram diferenças nas variáveis isocinéticas entre os membros inferiores, dominantes e não dominantes (CARVALHO; CABRI, 2007).

Durante cada teste, foi dado um encorajamento verbal e um feedback visual pela tela do computador, na tentativa de alcançar o nível de esforço máximo. Todos os procedimentos foram realizados pelo mesmo investigador.

Como medidas de respostas cardiovasculares, foram mensuradas a frequência cardíaca (FC, bpm) (PolarTM RS800CX, Kempele, Finlândia), a pressão arterial (PA, mmHg) e os índices de variabilidade da frequência cardíaca (VFC). A PA foi aferida de forma indireta pelo método auscultatório, sendo necessária a utilização de um esfigmomanômetro (Premium Aneroide Hospitalar, visor 15x15, São Paulo, Brasil) e um estetoscópio (3MTM Littmann Lightweight II, Neuss, Alemanha). Todas as aferições foram realizadas pelo mesmo pesquisador e em conformidade com as recomendações da Sociedade Brasileira de Cardiologia (VI DIRETRIZES BRASILEIRAS DE HIPERTENSÃO).

Assim que o voluntário chegava ao LEM - FEF/ UnB, era acoplada ao tórax dele a cinta do frequencímetro, modelo RS800CX (PolarTM, Kempele, Finlândia), logo abaixo da borda inferior do osso esterno, precisamente no processo xifoide do paciente, utilizando gel sob a fita para permitir melhor contato com a pele, para monitorização da FC e VFC.

Para mensuração da VFC pré-exercício, os voluntários foram orientados a permanecer sentados e sem conversar durante 15 minutos, já posicionados na cadeira do aparelho isocinético, precedido de um repouso absoluto de 10 minutos em ambiente calmo e silencioso. Já para mensuração da VFC pós-exercício, os voluntários foram orientados a permanecer na cadeira do aparelho isociné- tico por 5 minutos após a realização das 3 (três) séries do exercício. Para análise da VFC, foi utilizado um software (Kubios HRV analysis software, versão 2.0, University of Kuopio, Finlândia), que utiliza algoritmos específicos, como a transformada rápida de Fourier, para avaliação do espectro de potência da frequência cardíaca, onde as variações entre as baixas frequências $(\mathrm{BF})(0,04-0,15 \mathrm{~Hz})$ e altas frequências $(\mathrm{AF})(0,15-0,40 \mathrm{~Hz})$, bem como a sua relação, são indicadores das respostas autonômicas. Foram mensuradas as variáveis LF (ms2), [Banda de baixa frequência modulada pelo simpático e pelo parassimpático, com predominância simpática em algumas situações específicas, que reflete as oscilações do sistema baroreceptor], HF (ms2); [Banda de alta frequência que corresponde às variações da freqüência cardíaca relacionadas com o ciclo respiratório]; LF/HF (ms2), [Representa o balanço entre os sistemas simpático e parassimpático] e SD1 (ms), [Reflete a ação parassimpática].

A FC de pico obtida em cada série do exercício resistido foi visualizada por meio de gráficos gerados pelo software Polar pro Treiner, que acompanha o frequencímetro utilizado na pesquisa, sendo possível observar qual modalidade exigiu maior esforço miocárdico.

A PA foi aferida em 2 (dois) momentos: ao fim dos 15 minutos de repouso inicial, onde foi coletado o dado de VFC pré-exercício, e imediatamente após a realização da terceira série do exercício isocinético.

O tratamento estatístico dos dados foi realizado por meio do software GraphPad Prism $5^{\circledR}$ para Windows e planilha eletrônica do Excel. Primeiramente foi realizado teste Kolmogorov-Smirnov para verificar distribuição da população estudada. Posteriormente, foram utilizados os testes estatísticos 1 way ANOVA, com post-hoc Newman-keus, Teste t pareado e Wilcoxon, onde foi considerado como nível de significância estatística (p) um valor igual ou menor que 0,05 .

\section{Resultados}

A amostra foi composta de 13 indivíduos do sexo masculino, cujas características gerais da amostra são descritas na Tabela 1.

Tabela 1 - Características da amostra

\begin{tabular}{c|c|c|c}
\hline Idade (anos) & Peso (Kg) & Estatura (m) & IMC \\
\hline $28,40 \pm 6,10$ & $74,86 \pm 14,53$ & $1,78 \pm 0,07$ & $21,26 \pm 2,94$ \\
\hline
\end{tabular}


Em relação ao nível de atividade física, a amostra foi composta por cinco indivíduos muito ativos e oito ativos, segundo o IPAQ.

Quando comparados os valores de PAS pré e pósexercício, observaram-se diferenças significativas nas três modalidades, como mostra o Gráfico 1.

Gráfico 1 - Comparação entre PAS pré e pós-exercício, onde I, II e III - Séries dos Exercícios A1, A2 e A3, respectivamente. PAs i é a Pressão Sistólica Inicial (pré-exercício) e PAs f é a Pressão Sistólica Final (pós-exercício).

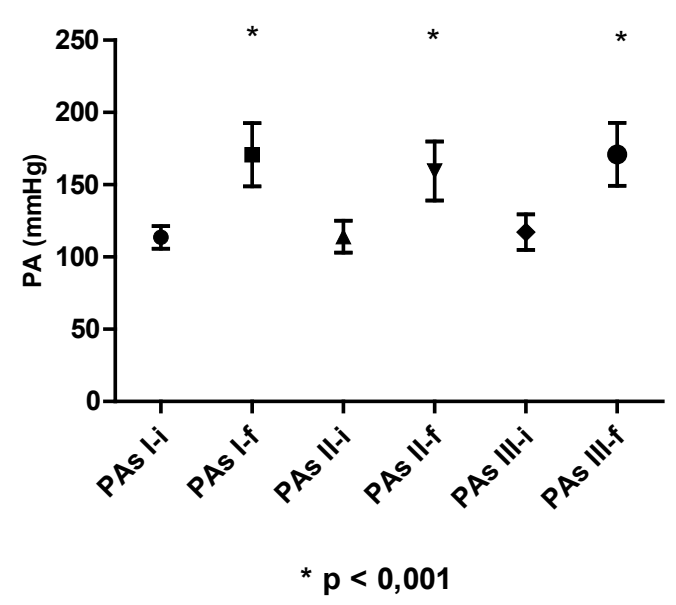

Em relação à $\mathrm{PAD}$ pré e pós-exercício, observouse diferenças significativas nas três modalidades, conforme Gráfico 2.

Gráfico 2 - Comparação entre PAD pré e pós-exercício, onde I, II e III são os Protocolos A1, A2 e A3, respectivamente. PAd i é a Pressão Diastólica Inicial (pré-exercício) e PAd f é a Pressão Diastólica Final (pós-exercício).

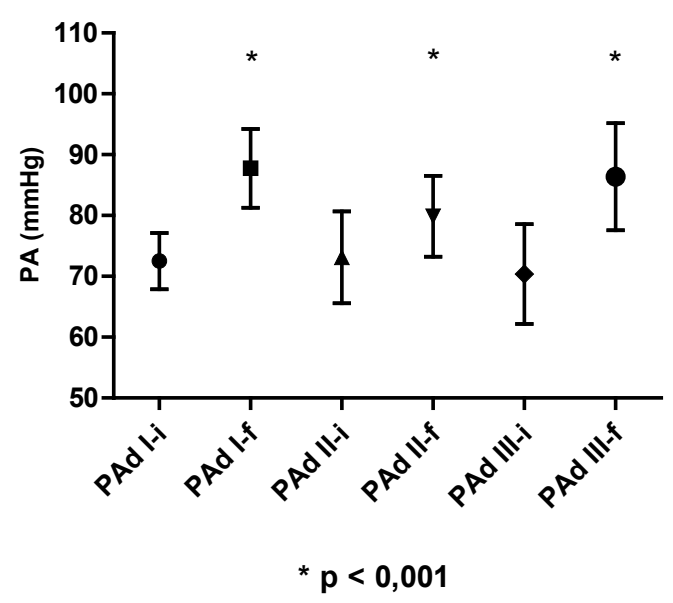

Quanto à FC, houve diferença significativa apenas entre a segunda e terceira série da modalidade A1 $(+4,28 \%)$. O gráfico 3 apresenta esses dados.
Gráfico 3 - Comparação entre as FC de pico de cada série, onde I, II e III é a séries dos Protocolos A1, A2 e A3, respectivamente. FC pico - Maior FC obtida em cada uma das 3 séries de cada protocolo.

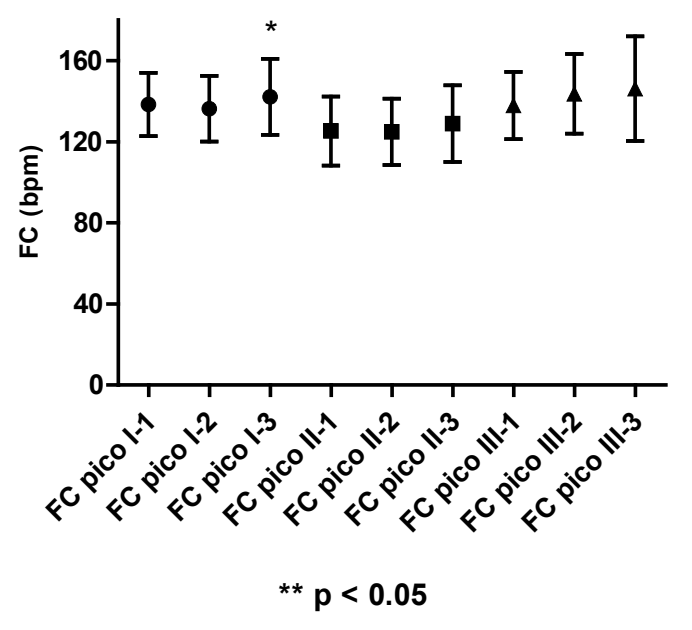

Como o teste de Kolmogorov-Smirnov apontou a VFC como uma variável não paramétrica, foram utilizadas Medianas 25\% percentil e 75\% percentil, para expressar as comparações feitas com essa variável.

Quando comparados os valores pré e pós-exercício do componente LF de cada modalidade, observaramse diferenças significativas nas modalidades A1 e A3, sendo mais significativo em A1, conforme gráfico 4 .

Gráfico 4 - Comparação entre as variações do LF pré e pósexercício.

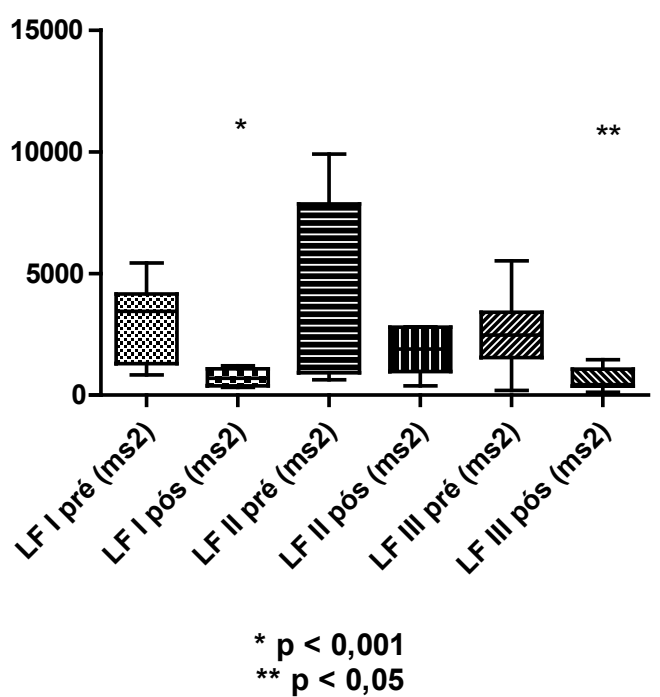

Quando comparados os valores pré e pós-exercício do componente HF de cada modalidade, observaram-se diferenças significativas nas modalidades A1 e A3, 
sendo mais significativas em $A 1$, enquanto $A 2$, em que $\mathrm{p}=0,08$ não demonstrou diferenças, conforme Gráfico 5 .

Gráfico 5 - Comparação entre as variações do HF pré e pósexercício

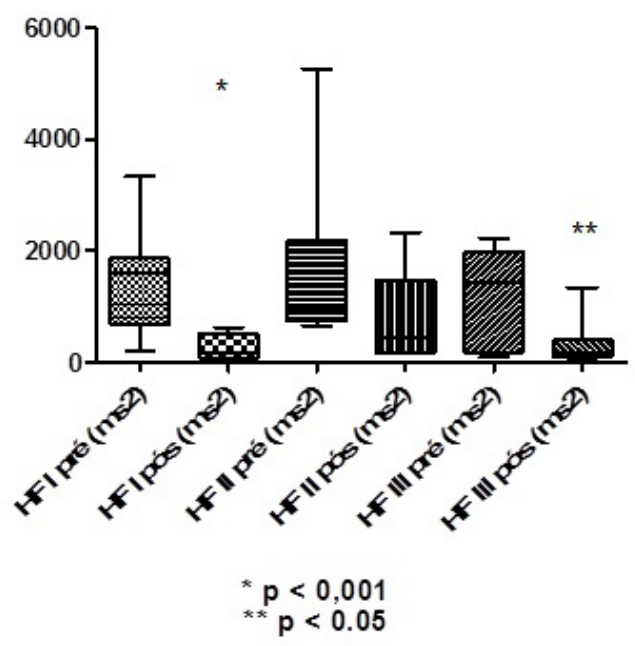

Não houve diferença estatisticamente significativa na relação LF/HF em nenhuma das 3 modalidades pré e pós-execução do exercício resistido, onde $\mathrm{p}=0,45 \mathrm{em} \mathrm{A1}$, $\mathrm{p}=0,56$ em A2 e p=0,47 em A3, como mostra o Gráfico 6 .

Gráfico 6 - Comparação entre as variações da relação LF/HF pré e pós-exercício

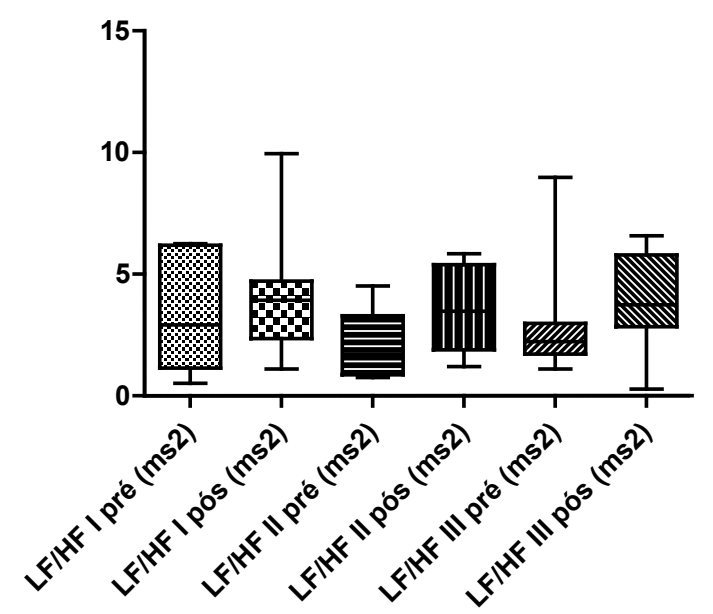

Para o SD1, houve diferença significativa pré e pós para a modalidade $A 1$, e uma tendência para as modalidades A2 e A3, onde $\mathrm{p}=0,07$ e 0,05, respectivamente, como mostra o Gráfico 7.
Gráfico 7 - Comparação entre as variações da SD1 pré e pósexercício

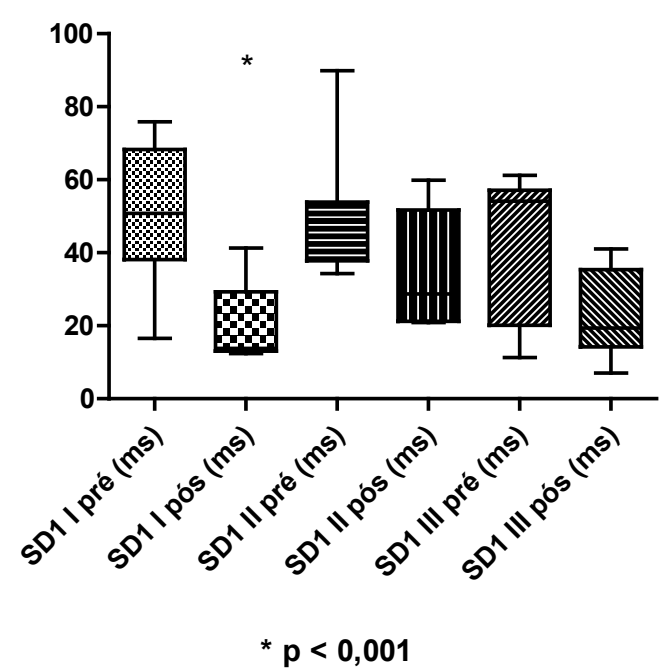

\section{Discussão}

Este estudo demonstrou alguns pontos importantes acerca dos ajustes cardiovasculares frente às diferentes metodologias de exercício resistido. Em relação à PA, foi constatada sobrecarga cardiovascular em todas as metodologias representadas pelos aumentos significativos da PA sistólica e diastólica, sendo estas mais exacerbadas em A1 e A3. A FC, apesar de ter apresentado um caráter ascendente entre as séries de todas as metodologias analisadas, demonstrou significância estatística apenas no aumento da segunda para a terceira série de A1. Quanto à VFC, notou-se que: a) a metodologia de exercício resistido concêntrico dos extensores do joelho (A1) gera maior stress cardiovascular quando comparado com os exercícios resistidos concêntricos dos flexores de joelho (A2) e exercício resistido alternado (A3); b) a metodologia A1 ocasiona maior estimulação autonômica, que parece ocorrer principalmente por retirada vagal.

Atualmente, a dinamometria isocinética é definida como padrão ouro para a avaliação da força e desempenho muscular (EVETCOVICH et al., 2001). O exercício resistido (ER) pode ser realizado em diversas modalidades, modificando a forma de ativação da muscu- 
latura agonista ou antagonista ao movimento solicitado (HASSANI et al., 2006). De forma geral, o ER promove uma sobrecarga cardiovascular que depende de um ajuste autonômico, refletindo em um aumento da resposta eferente vagal no controle do ritmo cardíaco (CARTER; BANISTER; BLABER, 2003). Os presentes resultados sugerem que mudanças autonômicas na variabilidade da frequência cardíaca podem ser induzidas por diferentes regimes de treinamento resistido.

Estudos prévios têm demonstrado que a resposta da FC aumenta consideravelmente com os exercícios resistidos, consequentemente, a PA acompanha tal ascendência (HEFFERMAN et al., 2006). Tal fato ocorre por aumentos na pressão intratorácica e redução no retorno venoso ocasionado por compressão vascular durante as contrações musculares (PAGANI et al., 1986). Segundo Heffernan et al. (2006), o aumento da FC ocorre inicialmente por inibição da estimulação parassimpática, como ficou evidenciado em nosso estudo pela redução do HF. Javorka et al. (2005) demonstraram que a modulação vagal não tinha recuperado 30 minutos após um exercício resistido de moderada intensidade, também evidenciada pela redução do HF power. Resultados similares, com 10 a 20 minutos após o exercício, foram demonstrados por outros autores (GALLAGHER; TERENZI; MEERSMAN, 1992; JAE et al., 2008). Segundo Kannankeril et al. (2004), essa redução do tônus vagal pós-exercício resistido pode ser explicada por alguns fatores, tais como: mudanças no volume plasmático sanguíneo, alterações na sensibilidade barorreflexa, alterações na modulação metabarorreflexa do tônus vagal e variações no volume corrente durante a execução do exercício, gerando alterações no retorno venoso e no estímulo ao nodo sinoatrial.

Em nosso estudo, encontramos, ainda que sem diferença estatisticamente significativa, um aumento na relação LF/HF, que tem sido empregada para avaliação do balanço autonômico entre os sistemas simpático e parassimpático (HEFFERMAN et al., 2006). Alguns estudos demonstraram que essa relação tende a permanecer alta por 30 a 60 minutos após uma sessão de exercício resistido (NOBREGA; ARAUJO, 1993; PIERPONT; STOLPMAN; GORNICK, 2000). Esse aumento na relação indica uma possível inibição parassimpática, entretanto, com exceção da metodologia A2, houve uma diminuição significativa da atividade simpática em nosso estudo, evidenciada pelo índice LF. Como o indicador de ação parassimpática HF também apresentou um comportamento descendente após a aplicação do ER, sugere-se que essa diminuição foi mais acentuada que em LF, justificando o discreto, mas perceptível, aumento na relação LF/HF.

Essa inibição parassimpática pós-exercício resistido pode ser explicada por duas teorias: comando central e o mecanorreflexo muscular (NOBREGA; ARAUJO, 1993). O comando central é um mecanismo de feed forward que, por meio de sinais oriundos de centros superiores, ativa o sistema cardiovascular e somático (WILLIAMSOM; FADEL; MITCHELL, 2006). O mecanorreflexo muscular acontece quando a contração do músculo esquelético ativo durante o exercício comprime mecanicamente fibras sensitivas aferentes, que podem gerar respostas de adaptações autonômicas (WILLIAMSOM; FADEL; MITCHELL, 2006). Araújo et al. (1992), demonstraram que a estimulação do mecanorreflexo por meio de cicloergômetro passivo de membros inferiores induziu a aumentos na FC, principalmente por retirada vagal. Como as modalidades de exercício resistido estimularam os mecanorreceptores musculares, isso pode ter levado à inibição parassimpática, principalmente na metodologia A1 (MAYNARD; EBBEN, 2003).

O nosso estudo apresentou limitações. Quando realizado um simples cálculo para mensurar o volume de exercício realizado pelos voluntários em cada metodologia de ER, notamos que em A3 esse valor representa o dobro das outras duas metodologias. Tal fato não representa grandes prejuízos, visto que, mesmo com o volume de exercício bem maior, tal metodologia não foi a que apresentou os valores estatísticos mais significantes. Assim, a dúvida que resta é se tal metodologia, se aplicada na mesma proporção das outras duas, apresentaria algumas significância estatística em relação aos ajustes cardiovasculares promovidos por ela.

Outro ponto a ser ressaltado é que o método auscultatório para aferição da PA, ainda que feita pelo mesmo pesquisador, é apontado por alguns autores como uma possível limitação para estudos experimentais. Segundo Wiecek et al. (1990), aferir a pressão arterial imediatamente após o exercício resistido, utilizando o método auscultatório, pode acarretar em erro de medida para baixo quando comparado com a medida de pressão arterial intra-arterial, tida como padrão ouro. Por outro lado, pela inviabilidade de se aferir a PA por meio de cateteres invasivos, as formas não invasivas de aferição da 
PA, ainda que menos fidedignas, são utilizadas em larga escala em ensaios clínicos, tornando-se dessa forma uma limitação relativa.

Contudo, este é um estudo inicial que nos mostra o comportamento cardiovascular frente a três diferentes metodologias de exercício resistido, o que, até então, é pouco descrito na literatura, de modo que outros estudos devem ser realizados com uma casuística maior e outras metodologias de ER.

De acordo com Umpierre e Stein (2007), o treinamento resistido tem sido proposto como uma possível estratégia para prevenção e reabilitação cardiovascular, o que o torna uma importante ferramenta clínica no acompanhamento de pacientes cardiopatas, devido à capacidade do exercício físico regular auxiliar no controle da PA em curto e longo prazo, em vista disso, é indicado como intervenção adjunta no manejo da hipertensão arterial. Embora os achados tenham mostrado uma sobrecarga imediatamente após a realização do ER, sabe-se hoje que, pouco depois disso, ocorre a hipotensão pós-exercício (HPE), que consiste na diminuição sustentada dos níveis pressóricos no pós-esforço. Uma das explicações para tal ocorrência é a de que o exercício físico promove, em longo prazo, o aumento das eferências parassimpáticas ao coração, tornando-o apto a ofertar a quantidade necessária de nutrientes ao organismo sem necessitar de tanto trabalho, o que infere na diminuição do débito cardíaco (DC) e, consequentemente, na diminuição da PA (PIERPONT; STOLPMAN; GORNICK, 2000; UMPIERRE; STEIN et al., 2007). Nessa tangente, o presente estudo se mostrou de grande valia ao indicar em qual tipo de exercício resistido, dos três analisados, a sobrecarga cardiovascular foi menos intensa, o que significa um risco menor ao paciente, ao mesmo tempo em que ele se beneficia dos efeitos dessa prática.

A redução do tônus vagal pode estar relacionada a uma maior propensão de doença cardíaca isquêmica, arritmias ventriculares e morte súbita após atividade física (GALLAGHER; TERENZI; MEERSMAN, 1992; KANNANKERIL et al., 2004; UMPIERRE; STEIN, 2007). Como a atividade parassimpática pode estar bem reduzida depois de uma sessão de exercício resistido, é razoável que consideremos o risco de alterações cardiovasculares após o exercício resistido em indivíduos com alterações cardiovasculares ou autonômicas.

\section{Conclusões}

As modalidades de exercício resistido apresentam efeitos distintos no sistema nervoso autônomo. A pesquisa indica que há uma redução no HF e um aumento na relação LF/HF nas modalidades diferenciadas, principalmente para o exercício concêntrico dos extensores do joelho. Essas alterações sugerem uma supressão vagal após uma sessão de exercício resistido. Futuros estudos deverão ser realizados para compreender mais acerca dos mecanismos de retirada de tônus vagal, assim como o possível risco cardiovascular relacionado à prática de exercícios resistidos mediante ajustes autonômicos.

\section{Referências}

ARAUJO, C. G. et al. Heart rate responses to deep breathing and 4-seconds of exercise before and after pharmacological blockade with atropine and propranolol. Clinical Autonomic Research, Oxford, v. 2, n. 1, p. 3540, feb. 1992. doi: 10.1007/BF01824209

ASSIS, M. M. V; OLIVEIRA, J. B. B. Medida indireta da pressão arterial: conhecimento teórico dos fisioterapeutas. Revista Brasileira em Promoção da Saúde, Fortaleza, v. 16, n. 1-2, p. 17-20, jan./dez. 2003.

CAMBRI, L.T. et al. Variabilidade da frequência cardíaca e controle metabólico. Arquivos Sanny de Pesquisa em Saúde, Santos, v.1, n.1, p. 72-82, set./out. 2008.

CARVALHO, P.; CABRI, J. Avaliação isocinética da força dos músculos da coxa de futebolistas. Revista Portuguesa de Fisioterapia no Desporto, Lisboa v.1, n. 2, p. 4-13, jul. 2007.

CARTER, J.B.; BANISTER, E. W.; BLABER, A. P. Effect of endurance exercise on autonomic control of heart rate. Sports Medicine, New Zealand, v. 33, n. 1, p. 33-46, jan. 2003. doi: $10.2165 / 00007256-200333010-00003$

EVETOVICH, T. K, et al. The effect of concentric isokinetic strength training of the quadriceps femoris on electromyography and muscle strength in the trained and untrained limb. The Journal of Strength \& Conditioning Research, Colorado, v. 15, n. 4, p. 439-445, nov. 2001.

FLECK, S. J.; KRAEMER, W. J. Fundamentos do treinamento de força muscular. 3. ed. Porto Alegre: Artmed, 2006.

FREEMAN, J. V. et al. Autonomic nervous system interaction with the cardiovascular system during exercise. Progress in Cardiovascular Disease, Philadelphia, v. 48 , n. 5 , p. $342-362$, mar./apr. 2006. doi:10.1016/j. pcad.2005.11.003 
GALLAGHER, D. ; TERENZI, T.; MEERSMAN, R. Heart rate variability in smokers, sedentary and aerobically fit individuals. Clinical Autonomic Research, Oxford, v. 2, n. 6, p. 383-387, dec. 1992. doi: 10.1007/BF01831395

HASSANI, A. et al. Agonist and antagonist muscle activation during maximal and submaximal isokinetic fatigue tests of the knee extensors. Journal of Electromyography and Kinesiology, New York v. 16, n. 6, p. 661-668, dec. 2006. doi:10.1016/j.jelekin.2005.11.006

HEFFERNAN, K. S. et al. Cardiac autonomic modulation during recovery from acute endurance versus resistance exercise. European Journal of Preventive Cardiology, London, v. 13, n. 1, p. 80-86, feb. 2006. doi: 10.1097/01. hjr.0000197470.74070.46

HEFFERNAN, K.S. et al. Heart rate recovery and heart rate complexity following resistance exercise training and detraining in young men. American Journal of Physiology. Heart and Circulatory Physiology, Bethesda, v. 293, n. 5, p. H3180-H3186, nov. 2007. doi: 10.1152/ajpheart.00648.2007

JAE, S. Y. et al. Slow heart rate recovery after exercise is associated with carotid atherosclerosis. Atherosclerosis, Amsterdam, v. 196, n. 1, p. 256-261, jan. 2008. doi: 10.1016/j.atherosclerosis.2006.10.023

JAVORKA, M. et al. Parasympathetic versus sympathetic control of the cardiovascular system in young patients with type 1 diabetes mellitus. Clinical Physiology and Functional Imaging, Oxford, v. 25, n. 5, p. 270-274, sep. 2005. doi: 10.1111/j.1475-097X.2005.00623.x

KANNANKERIL, P.J. et al. Parasympathetic effects on heart rate recovery after exercise. Journal of Investigative Medicine, Canada, v. 52, n. 6, p. 394-401, sep. 2004.

MALFATTI, C.A. et al. Análise da resposta da frequência cardíaca durante a realização de exercício isocinético excêntrico de grupamento extensor do joelho. Revista Brasileira de Fisioterapia, São Carlos, v. 10, n. 1, p. 5157, 2006. doi: 10.1590/S1413-35552006000100007

MAYNARD, J.; EBBEN, W.P. The effects of antagonist prefatigue on agonist torque and electromyography. The Journal of Strength \& Conditioning Research, Colorado, v. 17, n. 3, p. 469-474, aug. 2003.

NEVES, V. F. C. et al. Análises dos índices espectrais da variabilidade da frequência cardíaca em homens de meia idade e mulheres pós-menopausa. Revista Brasileira de Fisioterapia, São Carlos, v. 10, n. 4, p. 401-406, out./dez. 2006. doi: 10.1590/S1413-35552006000400007

NOBREGA, A.C. ; ARAUJO C.G. Heart rate transient at the onset of active and passive dynamic exercise. Medicine \&
Science in Sports \& Exercise, Wiscosin, v. 25, n. 1, p. 37-41, jan. 1993.

PAGANI, M. et al. Power spectral analysis of heart rate and arterial pressure variabilities as a marker of sympatho-vagal interaction in man and conscious dog. Circulation Research, Baltimore, v. 59, n. 2, p. 178-193, aug. 1986. doi: 10.1161/ 01.RES.59.2.178

PASCHOAL, M. A. et al. Variabilidade da frequência cardíaca em diferentes faixas etárias. Revista Brasileira de Fisioterapia, São Carlos, v.10, n. 4, p. 413-419, out./dez. 2006. doi: $10.1590 /$ S1413-35552006000400009

PIERPONT, G. L.; STOLPMAN, D. R.; GORNICK, C. C. Heart rate recovery post-exercise as an index of parasympathetic activity. Journal of the Autonomic Nervous System, Amsterdam, v. 80, n. 3, p. 169-174, may 2000. doi: 10.1016/S0165-1838(00)00090-4

POLITO, M. D.; FARINATTI, P. T. V. Considerações sobre a medida da pressão arterial em exercícios contrarresistência. Revista Brasileira de Medicina do Esporte, Niterói, v. 9, n. 1, p. 1-9, jan./fev. 2003. doi: 10.1590/S1517-86922003000100005

POLITO, M.D.; FARINATTI, P.T.V. Comportamento da pressão arterial após exercícios contrarresistência: uma revisão sistemática sobre variáveis determinantes e possíveis mecanismos. Revista Brasileira de Medicina do Esporte, Niterói, v. 12, n. 6, p. 386-392, nov./dez. 2006. doi: 10.1590/ S1517-86922006000600017

UMPIERRE, D. ; STEIN, R. Efeitos hemodinâmicos e vasculares do treinamento resistido: implicações na doença cardiovascular. Arquivos Brasileiros de Cardiologia, Rio de Janeiro, v. 89, n. 4, p. 256-262, out. 2007. doi: 10.1590/S0066782X2007001600008

VANDERLEI, L. C. M. et al. Noções básicas de variabilidade da frequência cardíaca e sua aplicabilidade clínica. Revista Brasileira de Cirurgia Cardiovascular, São José do Rio Preto, v. 24, n. 2, p. 205-217, abr./jun. 2009. doi: 10.1590/ S0102-76382009000200018

VIANNA, V.B.A. et al. Relação entre frequência cardíaca e consumo de oxigênio durante uma aula de "step training". Revista Brasileira de Ciência e Movimento, Brasília, v. 13, n. 1, p. 29-36, abr./jun. 2005.

WIECEK, E. M; MCCARTNEY, N; MCKELVIE, R. S. Comparison of direct and indirect measures of systemic arterial pressure during weightlifting in coronary artery disease. The American Journal of Cardiology, New York, v. 66 , n. 15 , p. $1065-1069$, nov. 1990. doi: 10.1016/00029149(90)90506-V

WILLIAMSOM, J. W.; FADEL, P. J.; MITCHELL, J. H. New insights into central cardiovascular control 
during exercise in humans: a central command update.

Experimental Physiology, New York, v. 91, n. 1, p. 51-58,

jan. 2006. doi: 10.1113/expphysiol.2005.032037 